\title{
Research on the Risk Factors and Countermeasures for Hospitalized Patients with Neurology
}

\author{
Xin Sui \\ The Third Affiliated Hospital of Qiqihar Medical University; Heilongjiang Qiqihar 161000, China
}

Keywords: risk factors; hospitalized patients; neurology

\begin{abstract}
To investigate the incidence of nosocomial infection in neurology and its related risk factors, and provide evidence for the prevention of nosocomial infection. The clinical data of 863 inpatients in Department of Neurology in our hospital from January 2013 to February 2015 were retrospectively analyzed to analyze the incidence of nosocomial infection, the site of infection, and the risk factors for infection. There were 42 cases of nosocomial infection in 863 patients, and the infection rate was $4.87 \%$. The most common infection sites were lower respiratory tract, urinary system, gastrointestinal tract, upper respiratory tract, and skin tissue, accounting for 42.85\%, $30.95 \%$, and $14.26 \%$, respectively. , $4.76 \%, 2.38 \%$, etc.; age $\geq 60$ years old, alcohol and tobacco history, hospitalization time $\geq 3 \mathrm{~d}$, use of antibiotics $>3 \mathrm{~d}$, tracheal intubation, mechanical ventilation, indwelling catheter are risk factors for nosocomial infection in neurology department. The incidence of nosocomial infection in patients with neurology is relatively high. The following respiratory tract, urinary system and gastrointestinal tract are common. The risk factors for nosocomial infection are relatively numerous and complex. The hospital needs to carry out targeted interventions to reduce the risk factors of nosocomial infection and the incidence of nosocomial infections.
\end{abstract}

\section{Introduction}

Neurology is an important clinical department for the treatment of critically ill neurological patients. The patients are generally older and are often applied to invasive procedures during rescue treatment. In addition, patients often have varying degrees of dyskinesia. Consciousness disorders, slower recovery of illness and longer hospital stay, poor treatment compliance, weak surgical tolerance, leading to a higher incidence of nosocomial infections in the neurology department than other departments in the hospital, and inpatients in neurology Both the therapeutic effect and the rehabilitation process will have a serious impact. In order to further reduce the probability of nosocomial infection in our department, we selected 180 neurology hospitalized patients and conducted the following process to in-depth analyze the risk factors of hospital infection and related nursing measures in our hospital. A detailed report is described below.

\section{Materials and Methods}

2014-02-2015-12 Inpatients in our department of neurology have 67 infections, including cerebral hemorrhage, cerebral infarction, subarachnoid hemorrhage, Girard-Barran syndrome, central nervous system infection, motor neuron disease, myasthenia gravis, etc., see Table 1 . The hospitalization time was 5 to 30 days, with an average of 13.5 days. The patient complied with the Diagnostic Criteria for Nosocomial Infection [1] and was admitted for no more than 48 hours. Among 950 patients, 138 had intracerebral hemorrhage, 14 had infection, and $10.11 \%$ had infection rate; 464 had cerebral infarction, 28 had infection, and $6.03 \%$ had infection rate; 80 had subarachnoid hemorrhage and 8 had infection. 10.00\%; 84 cases of central nervous system infection, 6 cases of infection, infection rate of $7.10 \%$; 72 cases of Girard-Barran syndrome, 6 cases of infection, infection rate of 8.33\%; 64 cases of multiple sclerosis, The infection rate was $4.68 \%$ in 3 cases, 48 cases in Parkinson's disease and 2 cases in infection rate $4.16 \%$. 


\section{Factor Analysis}

Inpatients of neurology department were mainly infected with 35 cases of respiratory tract infections, accounting for 52.23\%; 14 cases of upper respiratory tract, accounting for 20.90\%; 12 cases of urinary system, accounting for $17.91 \%$; 6 cases accounted for $8.96 \%$.

Nosocomial infection-associated risk factors Analysis age $>60$ years old, having a bad history of tobacco and alcohol, having diabetes and chronic underlying diseases (hypertension, coronary heart disease), hospitalization time $>2$ weeks, invasive procedures, disturbance of consciousness, and long-term use of glucocorticoids Hormones, these are positively related to the incidence of nosocomial infections.

Distribution of pathogens 162 strains of bacteria were cultured, including 98 strains of Gram-negative bacteria, accounting for 60.49\%; 46 strains of Gram-positive bacteria, accounting for $28.40 \%$; and 18 strains of fungi, accounting for $11.11 \%$. Pathogens are mainly Gram-negative bacilli.

\section{Results Discussion}

The condition of nervous system diseases is complicated. A considerable number of patients are critically ill, have long hospital stays, and have more time in bed, which greatly increases the chance of infection and affects the prognosis of patients. Therefore, it is important to understand the risk factors of infection and actively take preventive measures to restore the patient's condition and improve the prognosis. 3.1 Relationship between Inpatients and Diseases in Department of Neurology In this study, 67 patients were infected in 950 hospitals and the infection rate was 7.05\%, which is consistent with relevant literature reports [2]. Among the disease types of inpatients, the highest infection rate of cerebrovascular disease is. This may be related to the acute onset of such diseases and the need to stay in bed for a long time. Such people should pay attention.

Infectious site distribution of neurology hospitalized patients In this study, hospitalized patients were mainly infected with lower respiratory tract infection, accounting for $52.23 \%$, followed by urinary tract, upper respiratory tract, gastrointestinal tract, and shallow tissue. Respiratory tract infections are more frequent due to decreased cough reflexes and obstructed discharge of tracheal secretions. Combined with tracheotomy, endotracheal intubation, and the use of ventilators, respiratory mucosa is damaged and infection is also easily caused. Urinary tract infections are related to the long-term insertion of urinary catheters. Frequently placed urinary catheters easily damage the urethral mucosa, destroy the natural defense barrier of the urethra, and the bleeding caused by mucosal injury creates a suitable environment for the growth of bacteria.

Analysis of Infection Risk Factors of Inpatients in Department of Neurology (1) Age: This study shows that age $>60$ years old is susceptible to infection, as the elderly with aging, organ aging, decreased tissue function, and systemic and local immune function Decline. When suffering from a disease, its physical condition is even weaker and its resistance is poor. It can easily be infected by various germs or viruses. (2) Basic diseases: The function of the organs and tissues of the elderly is reduced. When there are complications, the burden on the body is heavier and the resistance is worse. (3) Invasive operations: Traumatic, not only will significantly reduce the patient's resistance, destroy the patient's normal mucosal defense system, but also allow the patient to increase access to external media. (4) The use of antibacterial drugs: In particular, the use of large amounts or multiple antibacterial drugs and glucocorticoids will not only make the patient's normal flora imbalance, but also increase the patient's drug resistance and increase the risk of nosocomial infection. (5) Hospitalization time: The results of this study suggest that if the patient stays in hospital for more than 2 weeks, the psychological burden will increase, resulting in bad mood, which not only affects the therapeutic effect, but also increases the chance of contact with pathogenic bacteria. (6) Disturbance of consciousness: coma patients have long-term bed rest, cough reflexes disappear, and it is difficult to eliminate respiratory secretions. In addition, invasive procedures, nutritional deficiencies and other factors increase the chance of hospital infection.

The type of disease with the highest infection rate in this study was cerebral hemorrhage, the 
infection rate was $7.69 \%$, and the cerebral infarction was the second, the infection rate was $6.0 \%$. The neurology department hospitalized patients may have obtained the above results under the combined effects of various factors. In the case of neurology, patients are generally older and have complex and sudden onsets. The condition is more dangerous, the condition is slower to recover, the length of hospital stay is longer, there is consciousness disorder, and dysphagia symptoms, physical mobility is significantly reduced and the size Incontinence and other clinical characteristics [3], patients with severe illness will lose protective reflexes, so the chance of hospital infection will significantly increase. The site with the highest infection rate in this study was the respiratory tract, and the infection rate was 3.33\%. The underlying cause may be related to the weakening of the cough reflex, the obstruction of airway secretions, and the relatively easy existence of bacterial growth. In addition, the patient is in the process of treatment. The use of respiratory equipment will cause damage to the patient's airway mucosal barrier and direct communication of the airway with the outside world, thereby significantly increasing the probability of hospital infection. According to relevant literature, the cause of high incidence of urinary tract infection in patients may be disturbance of consciousness and incontinence in the patient. There is a certain correlation between the use of continuous indwelling catheters. According to related reports, the illnesses in winter and spring, the age of onset over 60 years old, the history of tobacco and alcohol, the history of diabetes, the length of hospital stay, the poor state of consciousness, the use of broad-spectrum antibiotics, mechanical ventilation, and indwelling urinary catheters are nerves. Internal medicine hospital infection risk factors. The high incidence of multiple diseases in the winter and spring seasons, the hospital infection rate in hospitalized patients with neurology may be due to the colder winter and spring season, which reduces the respiratory mucosal defense and purification of patients, making it easier for bacteria and viruses to invade the human body. Infection rates have remained high; older people and patients with a history of tobacco and alcohol and a history of diabetes generally have poor immune function and are prone to infection; patients with longer hospital stays will have an increase in the number of treatments and invasive procedures, and therefore occur. There is a high incidence of nosocomial infections; patients with unconsciousness have unstable water and electrolyte balances, acid-base imbalances, and unstable internal conditions can cause patients to suffer from various complications due to stress reactions resulting in decreased anti-infective capacity. As a result, symptoms of infection appear; unreasonable use of antibiotics can lead to increased drug resistance and dysbacteriosis [4], which in turn can significantly increase the chance of hospital infection; tracheotomy and invasive mechanical ventilation can all lead to impairment of mucosal function. , hospital infection increased. Based on the above conditions, our hospital needs to carry out an in-depth analysis of the risk factors of nosocomial infection in inpatients in neurology department and give relevant nursing measures to significantly reduce the probability of patients with nosocomial infection. After a lot of practice and statistics, the specific nursing strategies are as follows: 1 Provide patients with a clean, well-ventilated ward environment, give the patient a daily cleaning and disinfecting room, and regularly provide indoor air culture monitoring; 2 through training, significantly improve the professional quality of medical staff and effectively cultivate the sterile concept of medical staff. Strict implementation of the hospital's various rules and regulations for the prevention of infection and strictly follow the aseptic process to operate, try to avoid giving patients invasive operation and disinfection work related articles; 3 on the patient's primary disease and underlying disease to give positive symptomatic treatment And closely monitor the patient's condition changes, by giving patients related care measures to encourage patients, improve patient compliance and immunity, reasonable use of antibiotics to achieve effective prevention and effective treatment.

Preventive Measures In 2016, the neurological department of our hospital treated patients with intensive care and established an intensive care unit to strengthen prevention and control measures for hospital infections: (1) Formulate disinfection and isolation systems and standards and ensure strict compliance with requirements. (2) Strengthen ward management, control external personnel to frequent the ward and close contact with the patient, often open the window for ventilation and daily disinfection. (3) Strengthen nursing, take care of people when necessary, and also strengthen 
life and psychological care for patients. A reasonable diet, more communication with patients, increase their confidence in overcoming the disease, so that it actively cooperate with the treatment. (4) Strictly control indications of invasive treatment, minimize trauma to elderly patients, and strictly perform aseptic procedures when invasive procedures are necessary. (5) The rational use of antibacterial drugs, as far as possible according to drug susceptibility testing for patients to choose the appropriate short-term antibacterial drugs [5]. (6) Medical staff use ventilators, central venous catheters, and indwelling catheters to evaluate their weaning and extubation daily to reduce the risk of infection. (7) The condition allows patients to raise their bed heads by $30^{\circ}$. Through analysis, it was found that implementation of the above measures greatly reduced the infection rate of hospitalized patients in our department of neurology.

\section{Conclusion}

For the high risk factors of nosocomial infection in inpatients in neurology department, we take active and effective preventive measures, which can greatly reduce the infection rate of hospitalized patients.

\section{References}

[1] Hidron AI,Edwards JR,Patel J,et al. NHSN annual update: antimicrobial-resistant pathogens associated with healthcare-associated infections: annual summary of data reported to the National Healthcare Safety Network at the Centers for Disease Control and Prevention,2006-2007[J].Infect Control Hosp Epidemiol,2008,29(11):996-1011.

[2] George J, Alangaden MD. Nosocomial Fungal Infections: Epidemiology, Infection Control, and Prevention [J].Infect Dis Clin N Am, 2011, 25(1):201-225.

[3] Bereket, Hemalatha, Getenet, et al. Upadate on bacterial nosocomial infections [J].European Review for Medical and Pharmacological Sciences, 2012, 16(8):1039-1044.

[4] Vazquez E,Murcia J,Canteras M,et al. Influence of a hygiene promotion programme on infection control in an intensive-care unit[J].Clin MicrobiolInfect,2011,17(6):894-900.

[5] Irene S. Kourbeti, John A. Papadakis, Christodoulos Neophytou, et al. Infections in patients with traumatic brain injury who undergoneurosurgery [J]. British journal of neurosurgery, 2011, 25(1):9-15. 